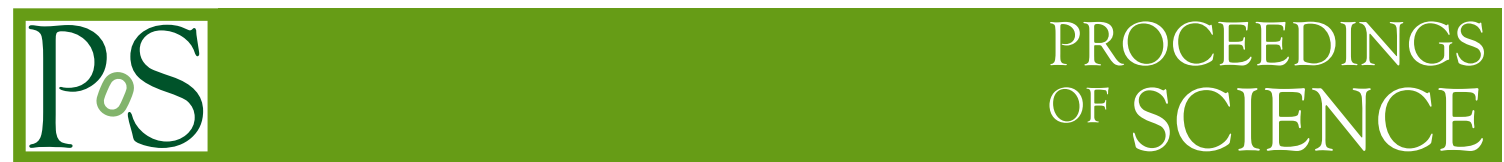

\title{
KM3NeT: Perspectives
}

\section{M. de Jong ${ }^{* \dagger}$}

Nikhef, Amsterdam, The Netherlands; and

Leiden Institute of Physics, Leiden University, Leiden, The Netherlands.

E-Mail: mjg@nikhef.nl

$\mathrm{KM} 3 \mathrm{NeT}$ is a large research infrastructure, that will consist of a network of deep-sea neutrino telescopes in the Mediterranean Sea. The main objectives of KM3NeT are the discovery and subsequent observation of high-energy neutrino sources in the Universe (ARCA) and the measurement of the mass hierarchy of neutrinos (ORCA). A cost effective technology for (very) large water Cherenkov detectors has been developed based on a new generation of low price 3-inch photo-multiplier tubes. Following the successful deployment and operation of two prototypes, the construction of the KM3NeT research infrastructure has started. The inprovements in the technology and the prospects of the different phases of the implementation of KM3NeT are summarised.

XVI International Workshop on Neutrino Telescopes,

2-6 March 2015

Palazzo Franchetti - Istituto Veneto, Venice, Italy

\footnotetext{
*Speaker.

On behalf of the KM3NeT Collaboration
} 


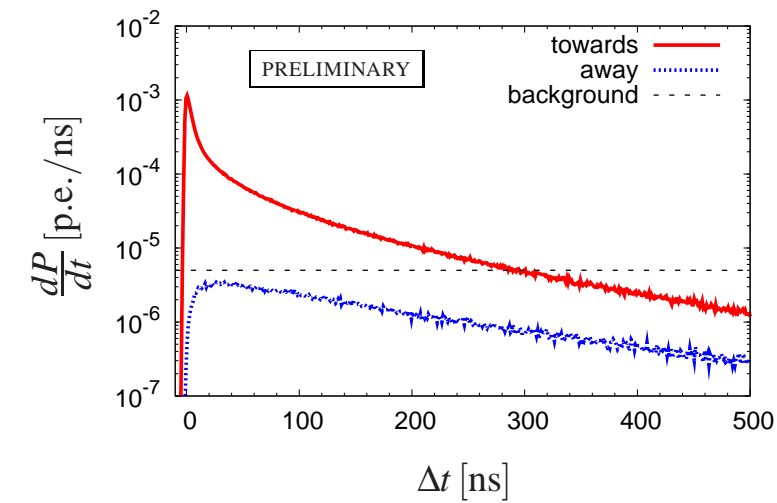

\section{Introduction} (ORCA) has separately been reported at this conference[4].

\section{Technology} an energy of $1 \mathrm{TeV}$ is shown in Figure 1.

The main objectives of KM3NeT are the discovery and subsequent observation of high-energy neutrino sources in the Universe (ARCA) and the measurement of the mass hierarchy of neutrinos (ORCA). The world-wide effort in this field is concentrated in three large research infrastructures, namely: IceCube[2] (South Pole), KM3NeT (Mediterranean Sea) and GVD[3] (Lake Baikal). The successful construction and operation of the ANTARES detector (Mediterranean Sea) has demonstrated the feasibility of deep-sea neutrino telescopes[5]. Furthermore, the transparency of the deep waters, the size of the detector and the geographical location make KM3NeT an ideal instrument to study sources of high-energy neutrinos in the Galaxy. The neutrino signal reported by IceCube supports the hypothesis that some astrophysical sources are capable of accelerating protons up to sufficient energies to trigger the production of (very) high-energy neutrinos[6]. The next challenge is to identify the sources of cosmic neutrinos. The measurement of the mass hierarchy of neutrinos

The detection principle is based on the detection of the Cherenkov light induced by relativistic charged particles emerging from an interaction of a neutrino in the vicinity of the detector. The angular resolution is primarily determined by the lever arm between the light sensors and the measurement precision of their positions and the arrival time of the Cherenkov light. Of the different types of neutrino interactions, the charged current interaction of a muon neutrino yields the best angular resolution because the muon that emerges from such an interaction has the longest range. The optical properties of the water can be summarised by the probability density function (PDF) of the arrival time of the Cherenkov light. As an example, the PDF corresponding to a muon with

Figure 1: The probability density function (PDF) of the arrival time of light from a $1 \mathrm{TeV}$ muon on a 3inch PMT. The left (right) figure is for a minimal distance of approach of 50 (100) $\mathrm{m}$. The red (blue) curve corresponds to a PMT with the front (back) facing the muon trajectory and the dashed horizontal line to the random background due to Potassium decays (and bioluminescence).

The light transmission properties of deep-sea water combined with the presently feasible position $(10 \mathrm{~cm})$ and time $(1 \mathrm{~ns})$ resolution of the PMTs make it possible to reconstruct the direction of high-energy muons with an accuracy of about 0.1 degrees. A new generation of 3 -inch 
photo-multiplier tubes (PMTs) has been developed for KM3NeT. These PMTs combine good timing (RMS less than 2 ns), relatively high quantum efficiency (up to 30\%) and low price (per unit photo-cathode area less than 10-inch PMTs).

The system provides sub-nanosecond precision on the measured time of single photo-electrons, while the position and orientation of the PMTs is measured to a few centimetres and few degrees, respectively. The PMTs and the readout electronics are hosted within pressure-resistant glass spheres, so called digital optical modules (DOMs). The DOMs are distributed in space along flexible strings, one end of which is fixed to the sea floor and the other end is held close to vertical by a buoy. The concept of strings is modular by design. The construction and operation of the research infrastructure thus allows for a phased and distributed implementation. A collection of strings form a single KM3NeT building block. The full KM3NeT telescope comprises six building blocks distributed on three sites, namely offshore Toulon (France), Capo Passero (Italy) and Pylos (Greece).

A building block comprises a large number of strings which are connected to junction boxes via interlink cables running along the seabed. Each string hosts a fixed number of optical modules which contain the PMTs. A main electro-optical cable connects the deep sea infrastructure to the shore station. The depth/distance of the infrastructure from shore are $2500 \mathrm{~m} / 50 \mathrm{~km}$, $3500 \mathrm{~m} / 100 \mathrm{~km}, 4500 \mathrm{~m} / 30 \mathrm{~km}$, for the Toulon, Capo Passero and Pylos site, respectively. It is foreseen that each site will host at least one building block. The site off shore Toulon will host the ORCA detector and the site off shore Capo Passero will host the ARCA detector. The detection efficiency of the ARCA detector has been studied as a function of $i$ ) the the number of strings, ii) the number of optical modules per string, ii) the horizontal spacing between strings and $i v$ ) the vertical spacing between optical modules for different absorption lengths of the water. In this, the efficiency is defined as the number of events due to an assumed flux of neutrinos from RXJ1713[8]. An optimum spacing has been found at about $90 \mathrm{~m}$ horizontal and $36 \mathrm{~m}$ vertical spacing. The number of events as a function of the number of strings and number of optical modules is shown in Figure 2. In this, the various detector configurations consistently comprise a total of 12,320 optical modules.
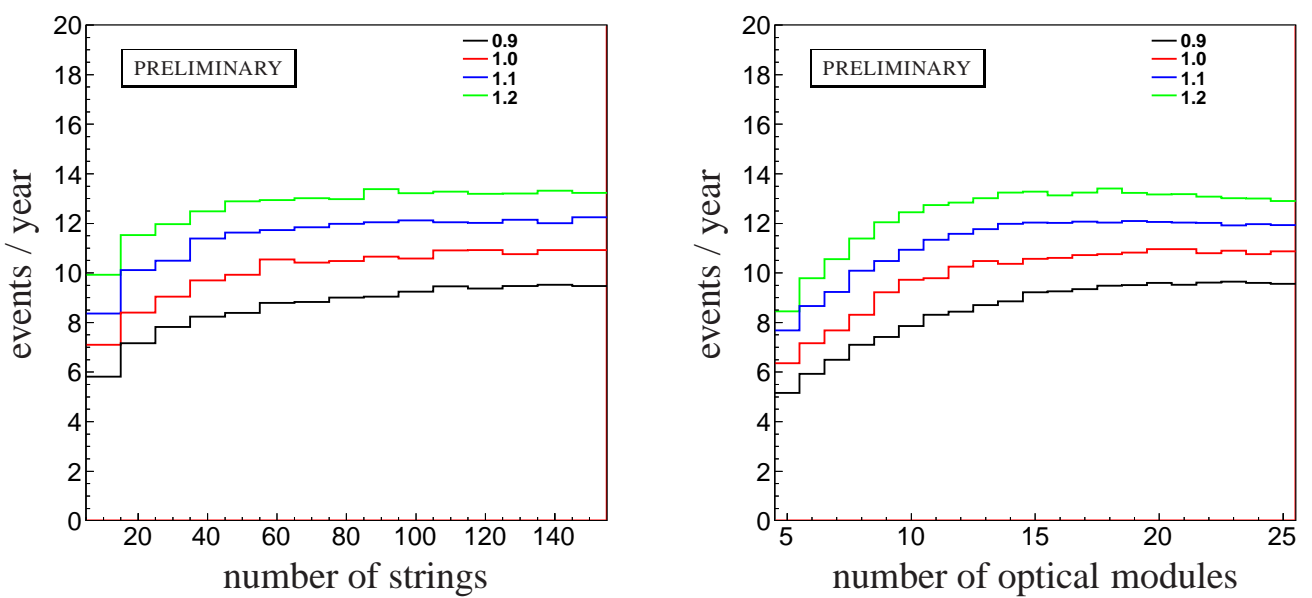

Figure 2: The number of events per year due to an assumed spectrum of neutrinos from RXJ1713 as a function of the number of strings and the number of optical modules per string. The neutrino spectrum is taken from reference[8]. The colour coding refers to a scaling factor applied to the absorption length. 
As can be seen from Figure 2, the normalised detection efficiency increases up to a point where it flattens out. The smallest detector with optimal efficiency corresponds to about 115 strings and 18 optical modules per string. This is referred to as a building block.

A string is about $700 \mathrm{~m}$ in length and hosts 18 optical modules spaced $36 \mathrm{~m}$ apart. It comprises two thin parallel ropes which hold the optical modules in place. Attached to the ropes is the vertical electro-optical cable ("backbone" cable), an oil-filled plastic tube which contains the electrical wires and optical fibres used for the power and data transmission. A surface boat will be used to deploy multiple strings in a single cruise. The strings are initially deployed on the seabed coiled around a spherical frame (so called launcher vehicle). A remotely operated vehicle (ROV) is used to deploy and connect the interlink cables from the base of a string to the junction box. The ROV also triggers the autonomous unfurling of a string after deployment.

A digital optical module is a transparent, pressure resistant, 17-inch glass sphere containing a total of 31 3-inch PMTs and their associated readout electronics. This design offers a number of improvements compared to previous designs based on a single large area PMT, most notably: Larger photo-cathode area, digital photon counting, directional information, wider field of view and reduced ageing effects. Furthermore, a position calibration device (acoustic piezo sensor) and a time calibration device (nano-beacon) are housed inside each glass sphere. The readout electronics feature low power consumption $(7 \mathrm{~W})$, high-bandwidth $(\mathrm{Gb} / \mathrm{s})$ data transmission using dense wavelength division multiplexing (DWDM) techniques, time over threshold measurement of each PMT signal and precision time synchronisation via the White Rabbit protocol. The optical module also incorporates an acoustic sensor and compass used for position and orientation calibration. A photo of an optical module is shown in Fig. 3.
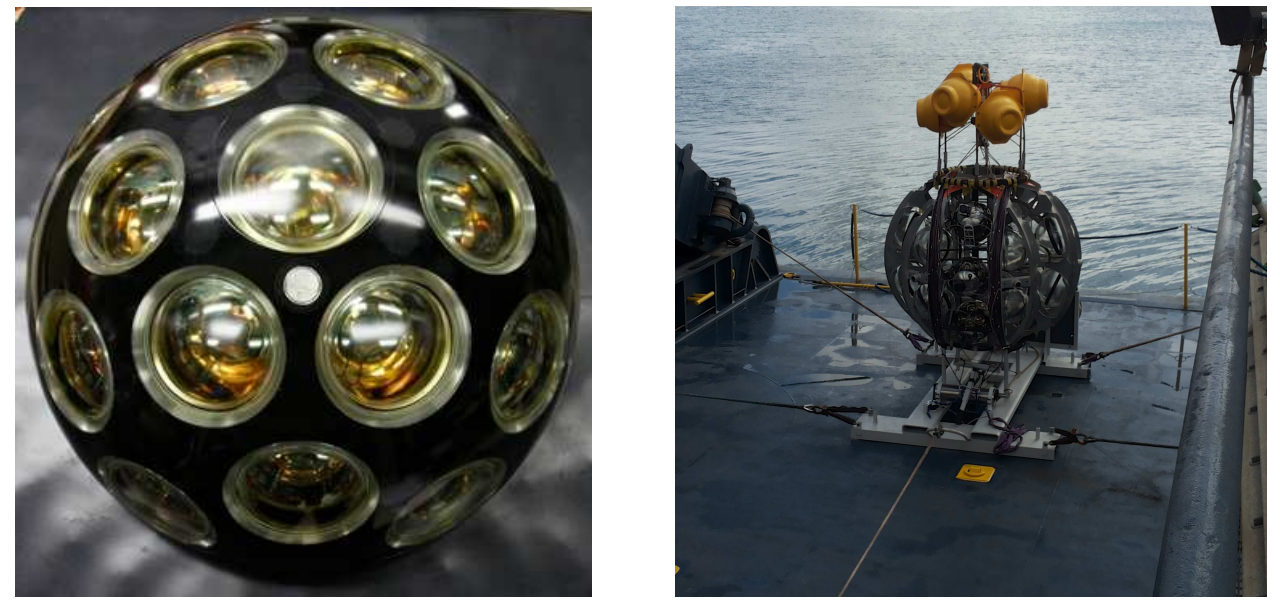

Figure 3: The KM3NeT optical module (left) consisting of a 17-inch pressure resistant glass sphere housing 31 3-inch PMTs. The small white dot in the middle is an acoustic piezo sensor which is used for position calibration. The prototype string (right) mounted on the launcher vehicle on board the Nautical Tide on its way from Malta to the deep-sea site, about 100 kilometres off the coast of Portopalo di Capo Passero, Italy.

A shore station is a (small) building which houses the equipment for GPS, power and real-time computing and a remotely operated control room. It provides a high-bandwidth Internet connection 


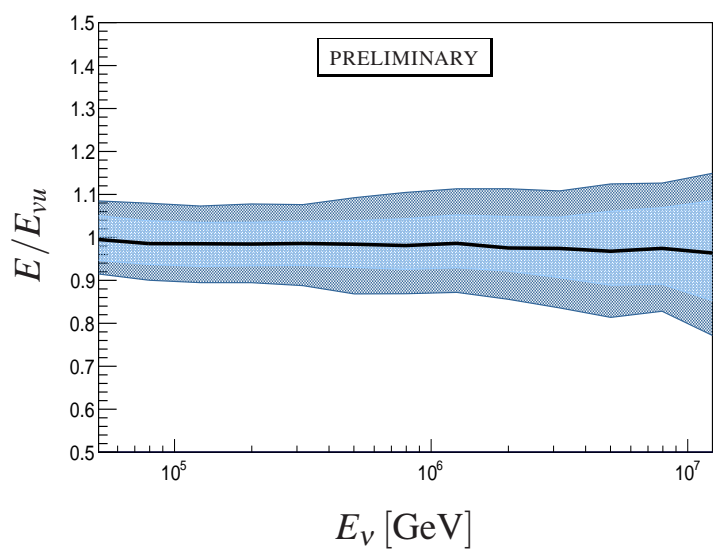
maintenance and security.

\section{Prospects}

to the central data repository. A crew of 2-3 persons is adequate for computer, network and power

A prototype of the KM3NeT optical module with 31 3-inch PMTs was mounted on the socalled instrumentation line of ANTARES and deployed in April 2013. The first results are published in reference[7]. This prototype optical module is still operational without any sign of degradation. In May 2014, a prototype string housing three optical modules was deployed offshore Portopalo di Capo Passero, Italy. A photo of this prototype string is shown in Fig. 3. A preliminary analysis of the first data confirms the specifications of the system[7]. Furthermore, evidence of correlated signals in the three optical modules from atmospheric muons has been found already.

The first observation by IceCube of extra-terrestrial neutrinos included a significant contribution of high-energy shower events[6]. These events are due to neutral current interactions of neutrinos or charged current interactions of electron neutrinos or tau neutrinos (in which the tau does not decay into a muon). Given the excellent angular resolution of the KM3NeT neutrino telescope for charged current interactions of muon neutrinos, the question then arises how well these events can be reconstructed. The energy resolution and the angular resolution of charged current electron neutrinos are shown in Figure 4. As can be seen from Figure 4, the energy resolution is about $10 \%$ and the angular resolution is about 2 degrees (the angular resolution is defined as the median space angle between the reconstructed direction and the true neutrino direction). This results makes it possible to do all flavour neutrino astronomy with ARCA.

Figure 4: The energy resolution (left) and the angular resolution (right) of charged current electron neutrinos as a function of the neutrino energy.

The construction and operation of the KM3NeT research infrastructure allows for a phased implementation. Recently, the KM3NeT collaboration started the first construction phase (phase1). Until 2016, 31 strings equipped with 558 optical modules will be assembled and deployed at the French and Italian sites. The resulting arrays will be different in size, the setup at the Italian site being significantly larger and providing the equivalent of about $10 \%$ of the size of the IceCube detector. The strings to be deployed at the Italian site will be configured for high-energy neutrino detection (ARCA) and the strings to be deployed at the French site for low-energy neutrino 
detection (ORCA). The ultimate goal is to fully develop the KM3NeT research infrastructure to comprise a distributed installation at the three foreseen sites (Italy, France and Greece), with almost 700 strings equipped with 12,400 optical modules in total (phase-2). The complete KM3NeT neutrino telescope will exceed the current IceCube detector by a substantial factor in sensitivity. The phase-2 detector will allow for $i$ ) an independent measurement of the IceCube signal with different methodology, improved resolution and complementary field of view, and ii) a timely measurement of the neutrino mass hierarchy. An overview of the prospectives of phase- 2 and phase- 3 is shown in Fig. 5.
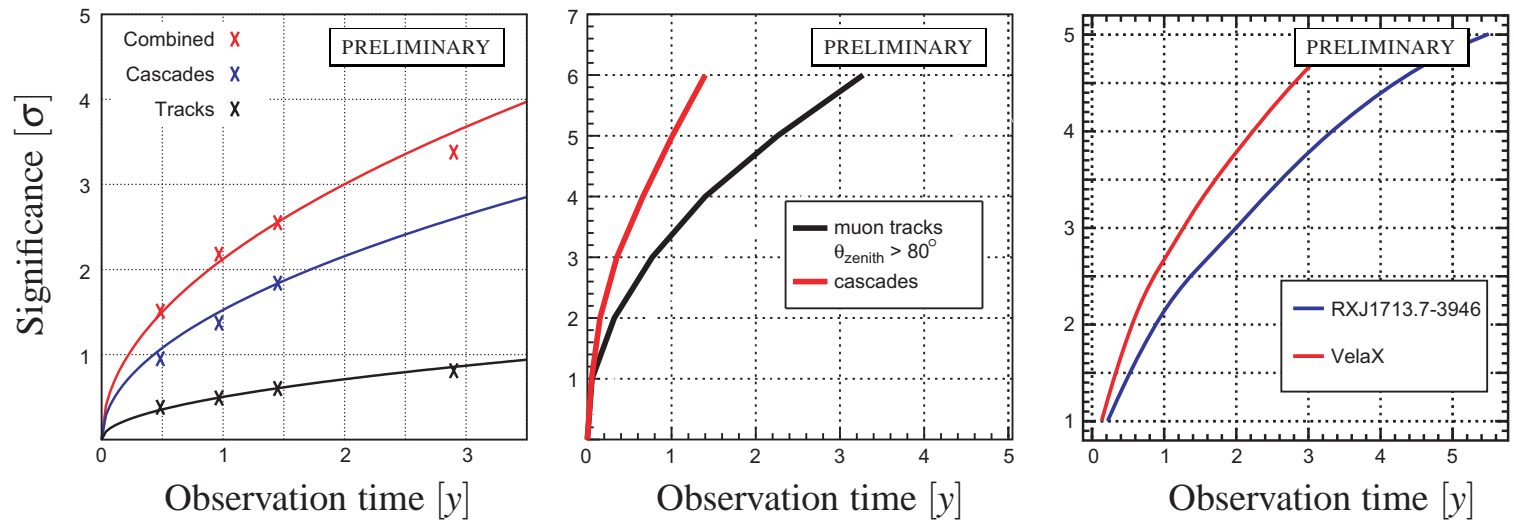

Figure 5: The significance $(\sigma)$ as a function of the observation time (years) for a measurement of the neutrino mass hierarchy using ORCA (left); the signal reported by IceCube[6] using ARCA (middle); and the assumed neutrino signals from the Supernova Remnant RXJ1713[8] and the Pulsar Wind Nebula Vela $\mathrm{X}[9]$ using KM3NeT phase-3 (right). 


\section{References}

[1] wWw. km3net.org

[2] icecube.wisc.edu

[3] baikalweb.jinr.ru

[4] See proceedings of this conference by T. Eberl.

[5] M. Ageron et al. [ANTARES Collaboration], Nucl. Instrum. Meth. A 656 (2011) 11

[6] M. G. Aartsen et al. [IceCube Collaboration], Science 342, 1242856 (2013)

[7] S. Adrián-Martínez et al. [KM3NeT Collaboration], Accepted for publication (EPJC-14-05-083.R1).

[8] S. R. Kelner, F. A. Aharonian and V. V. Bugayov, Phys. Rev. D 74 (2006) 034018 [Erratum-ibid. D 79 (2009) 039901]

[9] F. L. Villante and F. Vissani, Phys. Rev. D 78 (2008) 103007 